\title{
Estimation the Amount of Heat Generated by LEDs under Different Operating Conditions
}

\author{
Anna Andonova ${ }^{1}$, Namyong Kim², Nikolay Vakrilov ${ }^{1}$ \\ ${ }^{1}$ Department of Microelectronics, Technical University of Sofia, \\ Kl. Ohridski St. 8, BG-1979 Sofia, Bulgaria \\ ${ }^{2}$ Division of Electronic, Information and Communication Engineering, Kangwon National University, \\ Samcheok, South Korea \\ ava@ecad.tu-sofia.bg
}

\begin{abstract}
LEDs are not $100 \%$ efficient at converting input power to light. Some of the energy is converted into heat and must be transferred to the ambient. Two approaches are used to assess the amount of heat generated by the LED - based on the luminous efficacy of radiation and a new one presented in the paper. To provide sufficient accuracy in thermal evaluations closer to the real operating modes for a specific LED module a new technique is developed. The aim is to evaluate the amount of heat generated during design phase of LED lighting. Experiments of LED modules are conducted to evaluate LEDs efficiency at converting input power to heat. For the heat dissipation study and thermal analysis of LED module are used non-contact temperature measurement using an infrared camera combined with computer aided thermal modelling and simulation.
\end{abstract}

Index Terms-Design for quality; infrared imaging; light emitting diodes; thermal analysis.

\section{INTRODUCTION}

Luminous efficacy of LEDs that can be attained until 2015 is based on the expectation that LEDs will be able to attain $50 \%$ to $70 \%$ of the theoretical maximum. The theoretical maximum of the luminous efficacy is $300 \mathrm{~lm} / \mathrm{W}$, according J. Schmitz [1].

A key factor for LEDs is their efficiency of energy conversions. The main limitation to this energy conversion is a challenge for LED manufacturers and users [2].

The LED power conversion has two main constituent: electrical power-light conversion and electrical power-heat conversion. Other factors, such as driver efficiency, lighting optical losses, and lighting thermal factors will also determine the final energy efficiency of any LED lighting system [3].

The concrete value of the efficiency of LED system is related to the environment, operating mode and design [4], [5]. Thus, it must make compromises between cost, coolers, reliability and efficiency when designing LED lighting.

Performance estimation of LED from provided data by their manufacturers should be used only as a general indication and does not substitute detailed evaluation of individual LED. On the other hand "standard" or "reference" heat sinks do not exist for LEDs, complicating

Manuscript received 17 April, 2015; accepted 14 February, 2016. und.

This research was funded by a grant (DUNK-1/3) of Bulgarian Science their performance measurements [6].

Temperature variances determine the LED's efficiency, so thermal analysis and real operating tests are important approaches to design LED modules with long life.

Thermal analysis of white LEDs is still incomplete solved problem. Very often in the construction of LED lamps and lighting it cannot rely on sufficient and accurate data to determine the thermal performance of LEDs. In result an optimum selection of radiator do not ensure.

The amount of heat generated by a LED is often estimated by using a metric called Wall Plug Efficiency (WPE). WPE is dependent on the nominal flux and voltage, the actual drive current and junction temperature of the LED. Thus it is difficult to be defined a "typical" WPE value for accurate thermal evaluations at different operating conditions [7].

Another approach to assessing the amount of heat generated by the LED is based on the luminous efficacy of radiation (LER) [8]. The advantage of this approach is that LER value for an LED can be readily derived from the radiometric spectral power distribution, which is typically published in the LED datasheet. The analysis of LE in the phosphor-conversion white LED is not simple compared with the multi-chip-based white LEDs. Important problem is that do not available any study on the theoretical analysis of LER in the phosphor-conversion white LEDs in real working conditions [9].

This paper provides a new approach to estimate the amount of heat generated by LEDs in real operating conditions based on Computational Fluid Dynamics (CFD) simulation and thermographic Non-Destructive Evaluation (NDE). The method is applicable for single LED modules as well as for multi-chip LED modules. Thermal impact at different operating conditions has been analysed evaluating thermal power (i.e. how much power is converted to heat within the lighting source) by heat-dissipation coefficient for better structure design. On this way the design of LED modules can be optimized with respect to power-heat conversion through numerical simulation and temperature measurements.

\section{Method of Evaluation}

In order to study in detail and to assess the accuracy and applicability of our approach the following tools have been 
used:

- CFD thermal modelling and simulation [10];

- Physical experiment with contact and non-contact thermal measurements [11];

- Optical and electrical power measurements and calculation of junction temperature in operation [12].

Measurements based on two-test procedures described in [13] have been carried out with the cooperation of Octa Light AD. Measurements under different operating conditions were carried out on 50 samples with the same structure. In result it is obtained a mean value $K_{h d}=0.7$ for the working conditions reported in this paper. Same samples were measured by proposed new approach. It was found that the difference in the measured values of heat-dissipation coefficient by the two approaches does not exceed $5 \%$.

Basic steps of the proposed approach are:

- CFD simulations for different values of heat-dissipation coefficient;

- Temperature measurement by infrared camera;

- Depending on the specific design of the LED modules coordinates of characteristic informative points are selected (one to three) respectively the front and back side of the specimen. The simulated and the measured temperatures are compared for these points. There is a coincidence of the values whereupon they differ no more than $\pm 5 \%$;

- Calculate the average value measured for a minimum of three repetitions of the measurement;

- Determine at found accordance with what value of heatdissipation coefficient has been carried out the simulation and it is the sought value.

Three points, indicating the temperature of the sample during the simulation and measurement are selected respectively on the front (on side-diode) marked as F1, F2, F3 and on the back side marked as B1, B2 and B3. Points with the same numbers are one above the other. To reduce the influence of noise in the infrared image it is recommended to be used an average temperature of the temperatures of neighbouring pixels of the region of interest (ROI). ROI covers a $3 \times 3$ matrix, where the middle pixel is the selected one. In this case one can use a single couple temperature on the sample, rather than three.

The new approach allows more accurate adequacy evaluation of the simulation results, obtained by setting different working characteristics. Such evaluation is required to determine the results applicability from thermal modelling and simulation for new LED structure design with different topological configurations and implementation with various material systems. Hence, the best suitable LED module structure can be selected for the design of a particular lighting application.

\section{A. Measurement Setup}

LED source, based on Luxeon REBEL White, with $1 \mathrm{~W}$ power was selected for study [14]. The LED is mounted on specialized thick ceramic structure with ceramic padding and dimensions $4.61 \mathrm{~mm} \times 3.17 \mathrm{~mm} \times 0.63 \mathrm{~mm}$.

For assessing the thermal efficiency of the selected LED prototype a contactless temperature measurement by infrared camera ThermaCAM SC640 is used [15]. The thermal imaging camera has a thermal sensitivity less than
$0.05{ }^{\circ} \mathrm{C}$. The infrared resolution of the camera is $640 \times 480$ pixels. The images of the LED during steady state for $10 \mathrm{~min}$ were captured with rate of $30 \mathrm{fps}$.

LED module is placed in a thermostatically controlled box for maximum offset the effects of environmental on IR thermal measurement. To obtain results with high accuracy in IR thermography it is necessary to further measure emissivity, humidity and ambient temperature near the studied object.

A fine gauge wire thermocouple ( 36 gauge, $\mathrm{K}$ but $\mathrm{J}$ wire may also be implemented) is used to minimize the local cooling from the thermocouple during contact measurements. When the new method is applied by using a calibrated infrared camera, thermocouple is not used.

The results of contact and respectively contactless temperature measurements were no more than $2{ }^{\circ} \mathrm{C}$. The humidity is $40 \%$ and the ambient temperature is $22{ }^{\circ} \mathrm{C}$ during the IR thermography measurement of the LED module.

The principle of operation in conducting the physical experiment is illustrated on Fig. 1.
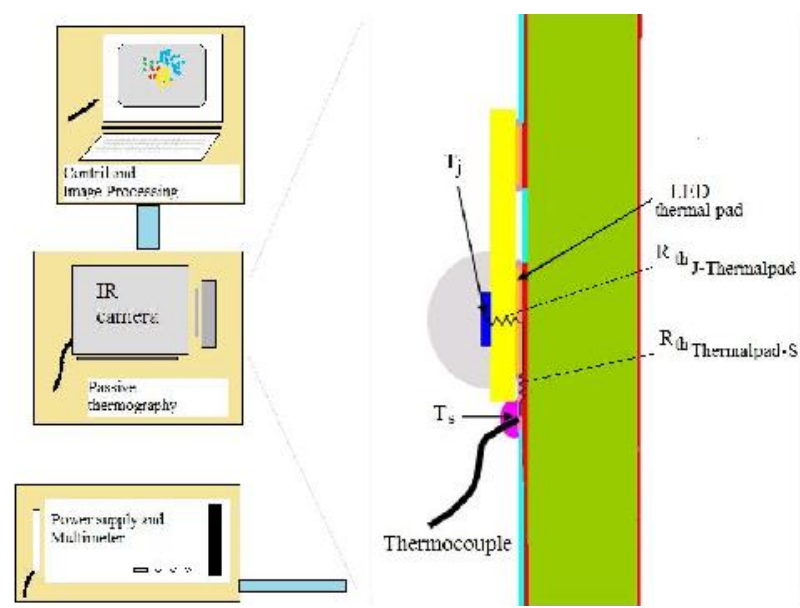

Fig. 1. Schematic diagram of the temperature measurement and analysis of results.

When LED module without additional cooling besides the ceramic board is studying, a low straight current value is used $-150 \mathrm{~mA}$ at a voltage of $2.8 \mathrm{~V}$, instead of the nominal value of $350 \mathrm{~mA}$ at a voltage $3.4 \mathrm{~V}$ in order not to chip overheat.

The measurements were carried out after establishing the dynamic temperature equilibrium - temperature change is less than $1{ }^{\circ} \mathrm{C}$ for 5 minutes. Figure 2 shows a frame, processed by the software ThermaCAM Researcher Professional v.2.9, for temperature variation at two points (SP05 and SP03). The distance from the measured LED module to the camera lens is $8 \mathrm{~cm}$ (close-up $50 \mu \mathrm{m}$ lens is used).

\section{B. Thermal CFD Modelling and Simulation}

For the creation of the computer 3D CFD model of the LED module the FLOTHERM environment is used [16]. A compact thermal model is built with next specifics:

- ceramic substrate from Alumina 96;

- printed AgPt (ESL 9562 G) layer;

- mechanical model of LED Luxeon REBEL White added from the site Philips [14] through the additional program FloBridge, with simplification level $50 \%$. 


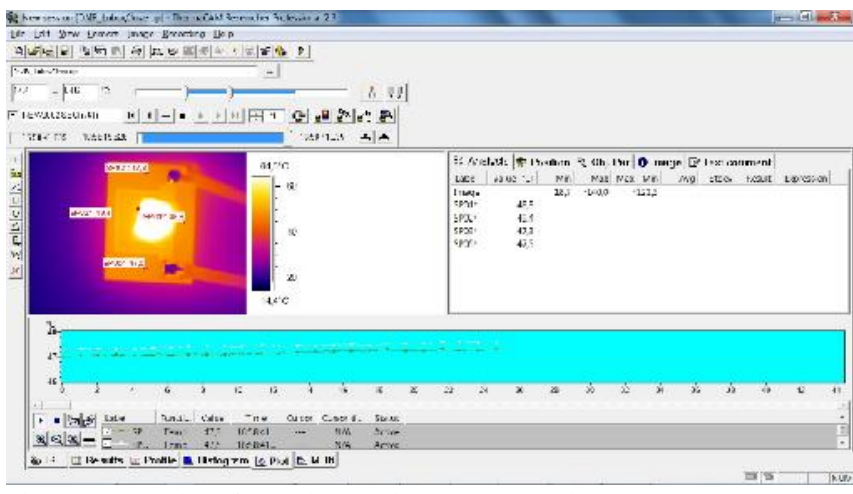

Fig. 2. Screenshot from the IR images processing program Researcher showing a frame of surface temperature measurement after calibration.

CFD simulations were carried out to determine more precisely what part of the input power is dissipated as heat. Several simulations are made with different values (from 0.65 to 1.0 ) of the coefficient of heat dissipation. The shown results in the paper are for electrical power of the LED $P_{d}=$ $U_{F} \times I_{F}=2.8 \mathrm{~V} \times 0.15 \mathrm{~A}=0.42 \mathrm{~W}$.

The heat-dissipation coefficient $K_{h}$ defines the portion of the total input LED electrical power that is dissipating as heat. It can be expressed as

$$
K_{h}=P_{h} / P_{e}=\left(P_{e}-P_{o}\right) / P_{e}=1-\eta_{W P E}
$$

Since $K_{h}$, can be obtained based on thermographic procedure, wall-plug efficiency $\eta_{W P E}$ can be calculated with the knowledge of $K_{h}$ using (1).

\section{RESULTS AND DISCUSSIONS}

\section{A. Results from IR-Camera Measurements}

IR-camera image is presented, from the back side of the examined module on Fig. 3.

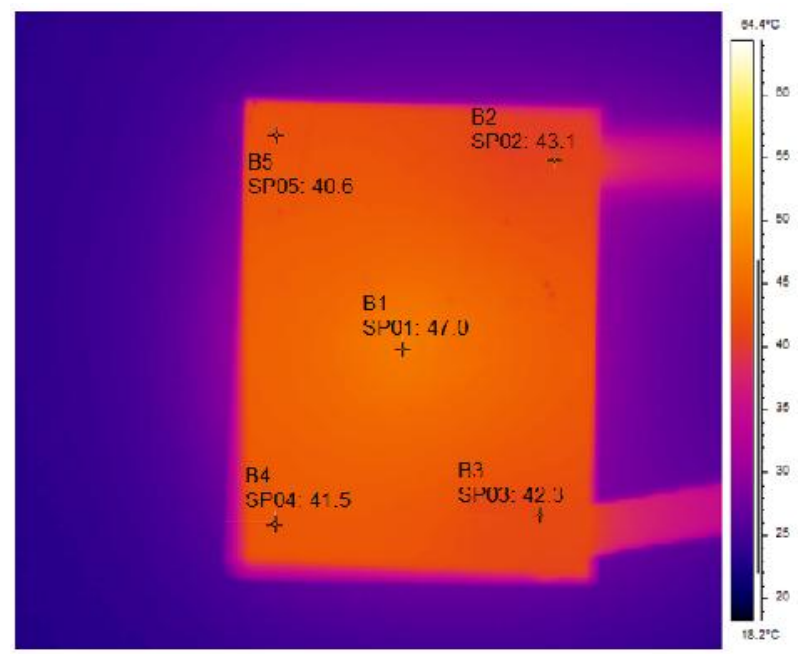

Fig. 3. IR image of the LED module's back side view.

Five points are chosen (marked from B1 to B5) on the thermal image for simulation verification and their temperature levels are shown.

A top-view of the diode thermogram is shown on the Fig. 4. The diode itself is covered and the metal layers of its structure are visible.

The temperature is measured in the same five points, resp. F1 to F5 on the front side of the LED module.
The temperatures measured on the bottom and front sides are given in Table I.

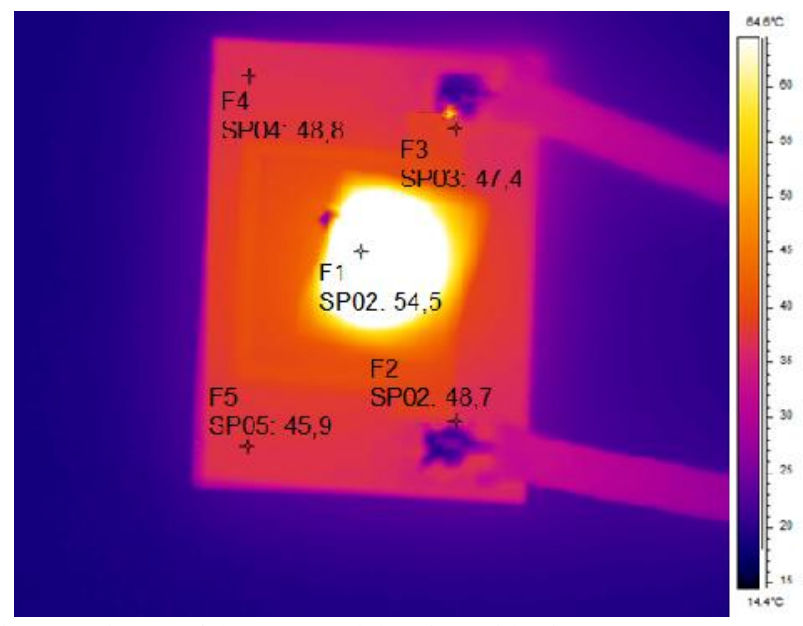

Fig. 4. IR image of the LED module's front side view.

TABLE I. TEMPERATURE DISTRIBUTION IN THE LED STRUCTRURE.

\begin{tabular}{|c|c|c|c|c|}
\hline \multicolumn{5}{|c|}{ LED module } \\
\hline \multicolumn{5}{|c|}{ Temperature in the points on the back side $\left[{ }^{\circ} \mathrm{C}\right]$} \\
\hline B1 & B2 & B3 & B4 & B5 \\
\hline 47,0 & 43,1 & 42,3 & 41,5 & 40,6 \\
\hline \multicolumn{5}{|c|}{ Temperature in the points on the front side $\left[{ }^{\circ} \mathrm{C}\right]$} \\
\hline 54,5 & F2 & F3 & F4 & F5 \\
\hline & 48,7 & 47,4 & 48,8 & 45,9 \\
\hline
\end{tabular}

To find the junction temperature we use the equation [17]

$$
\begin{gathered}
T_{j}=T_{r e f}+\left(R_{t h_{j-a}} \times P_{d}\right)= \\
=47^{\circ} \mathrm{C}+\left(11.72^{\circ} \mathrm{C} / \mathrm{W} \times 0.42 \mathrm{~W}\right) \approx 51.92^{\circ} \mathrm{C},
\end{gathered}
$$

where $T_{\text {ref }}$ is the LED's body temperature, measured via IR camera; $R_{t h ~}^{j-a}$ is the thermal resistance between the junction and the environment; $P_{d}$ is the dissipated thermal power.

We compute $R_{t h ~}^{j-a}$ as sequentially connected resistances, representing all layers in the way of heat transfer, from the source to the environment. The influence of the thinner layers which are not represented in the model can be omitted, as they are orders of magnitude less than $R_{t h j-a}$

$$
\begin{gathered}
R_{t h_{j-a}}=R_{t h_{j-c}}+R_{t h_{A l_{-} 96}}+R_{t h_{A g}}= \\
=10^{\circ} \mathrm{C} / \mathrm{W}+1.72{ }^{\circ} \mathrm{C} / \mathrm{W}+0.00816^{\circ} \mathrm{C} / \mathrm{W} \approx \\
\approx 11.72{ }^{\circ} \mathrm{C} / \mathrm{W},
\end{gathered}
$$

where $R_{t h j-c}$ is the typical thermal resistance from the junction to the heat drop, taken from specification of Luxeon REBEL White; $R_{r h}$ Al_ $_{-} 96$ is the thermal resistance of the ceramic substrate, which is computed using (3) below.

$R_{t h ~}$ g is the thermal resistance of the metal layer which is silver in our model and is computed again using

$$
R_{t h}=L / k A \text {, }
$$

where $L$ is layer's thickness; $k$ is thermal conductivity; $A$ is area.

The thickness $L$ of the substrate equals $630 \mu \mathrm{m}$, and for the silver metal layer equals $50 \mu \mathrm{m}$. The thermal 
conductivity $k$ of the ceramic substrate is $25^{\circ} \mathrm{C} / \mathrm{W}$, and for silver it is $419^{\circ} \mathrm{C} / \mathrm{W}$.

After substitution of the thermal resistance of the ceramic substrate, the thermal resistance of the silver layer in (3) we get respectively $R_{r h} \quad$ Al_96 $=1.72{ }^{\circ} \mathrm{C} / \mathrm{W}$ и $R_{t h} \quad$ Ag $=$ $0.00816^{\circ} \mathrm{C} / \mathrm{W}$.

\section{B. Results from the Thermal Simulations}

The result of the thermal simulation of the LED structure with power $0.42 \mathrm{~W}$ is presented on Fig. 4 . On the front side of the LED module the temperature markers are positioned in three points, where the temperatures are $F 1=50{ }^{\circ} \mathrm{C}$ и $F 2=48.8^{\circ} \mathrm{C}$ и $F 3=48.5^{\circ} \mathrm{C}$.
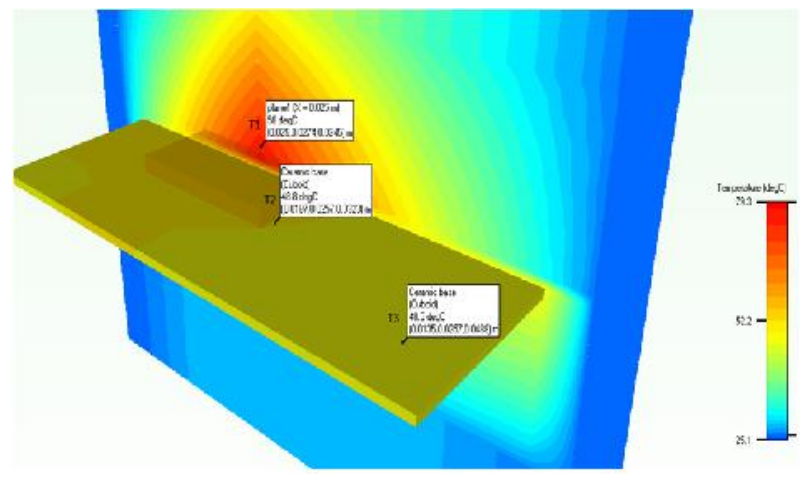

Fig. 4. Temperature distribution in the model of a LED module.

The results from thermal simulation at $K_{h}=0.85$ and $K_{h}=$ 0.90 on front side are shown in Fig. 5 and Fig. 6, respectively.

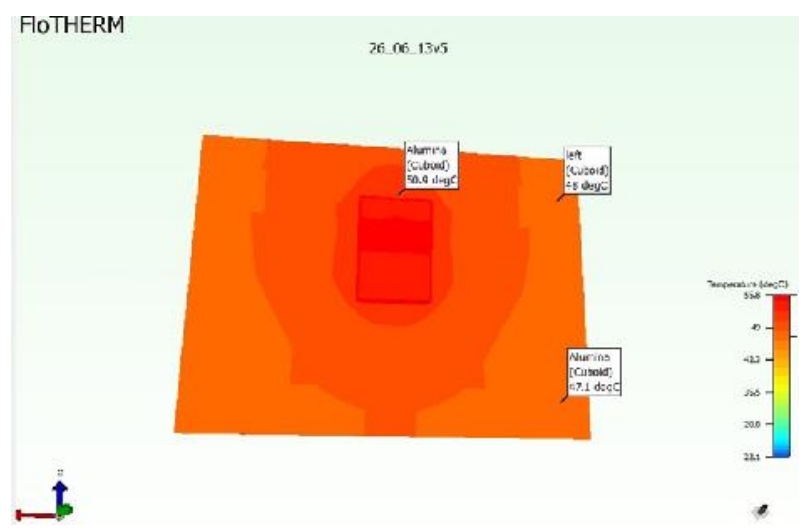

Fig. 5. Temperature distribution in the model of a LED at $K_{h}=0.85$.

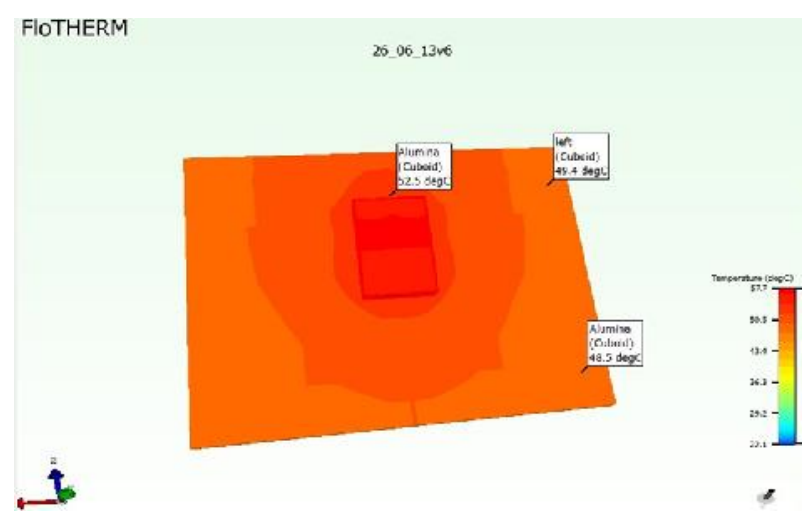

Fig. 6. Temperature distribution in the model of a LED at $K_{h}=0.90$.

We can see from the simulation results, that the temperatures of the ceramic substrate are quite close to those acquired via IR-thermography shown on Fig. 3 in points $\mathrm{F} 1=47.4{ }^{\circ} \mathrm{C}$ and $\mathrm{F} 2=47.7^{\circ} \mathrm{C}$. At environment temperature of $22{ }^{\circ} \mathrm{C}$, the relative error between measured and simulated temperatures in a given control point (in this case point $\mathrm{F} 1$ ) can be calculated with formula

$$
\begin{gathered}
\Delta T=\left(T_{I R}-T_{\text {sim }} / T_{I R}-T_{a}\right) \times 100= \\
=\left(47.4{ }^{\circ} \mathrm{C}-48.5^{\circ} \mathrm{C} / 47.4{ }^{\circ} \mathrm{C}-22^{\circ} \mathrm{C}\right) \times 100 \approx 4.01 \%,
\end{gathered}
$$

where $T_{I R}$ is IR thermography measured temperature; $T_{\text {sim }}$ is simulated temperature; $T_{a}$ is measured environment temperature during the physical experiment.

Optical evaluation of the value heat generated by the same LED module has been carried by using approach given in [8]. The value of $K_{h}=0.75$ has been got at the working mode in which the simulations and infrared measurements are performed. Temperature differences between simulation and IR-camera measurements at these working conditions are under $1.1{ }^{\circ} \mathrm{C}$, giving error of about $4 \%$.

In Table II the summary of the results from simulation of the LED structure are shown for different values of $K_{h}$ at the points used in measurements.

TABLE II. RESULTS FROM SIMULATIONS OF THE MODULE AT STEADY STATE.

\begin{tabular}{|c|c|c|c|c|c|c|}
\hline point & $\begin{array}{c}\mathbf{B 1} \\
{\left[{ }_{\boldsymbol{h}}{ }^{\circ} \mathbf{C}\right]}\end{array}$ & $\mathbf{B 2}\left[{ }^{\circ} \mathbf{C}\right]$ & $\begin{array}{c}\mathbf{B 3} \\
{\left[{ }^{\circ} \mathbf{C}\right]}\end{array}$ & $\begin{array}{c}\mathbf{F 1} \\
{\left[{ }^{\circ} \mathbf{C}\right]}\end{array}$ & $\begin{array}{c}\mathbf{F 2} \\
{\left[{ }^{\circ} \mathbf{C}\right]}\end{array}$ & $\begin{array}{c}\mathbf{F 3} \\
{\left[{ }^{\circ} \mathbf{C}\right]}\end{array}$ \\
\hline 0.65 & 44.9 & 42.7 & 42.6 & 46.9 & 44.3 & 42.9 \\
\hline 0.70 & 45.7 & 43.7 & 43.5 & 48.6 & 45.8 & 44.4 \\
\hline 0.75 & 46.5 & 44.6 & 44.4 & 50.3 & 47.3 & 45.7 \\
\hline 0.80 & 47.5 & 45.5 & 45.3 & 52.0 & 48.7 & 47.1 \\
\hline 0.85 & 48.1 & 46.4 & 46.2 & 53.6 & 50.2 & 48.5 \\
\hline 0.90 & 48.8 & 47.3 & 47.1 & 55.3 & 51.7 & 49.8 \\
\hline 0.95 & 49.4 & 48.1 & 47.8 & 57.4 & 52.8 & 50.8 \\
\hline 1.00 & 50 & 48.8 & 48.5 & 58.5 & 53.0 & 51.6 \\
\hline
\end{tabular}

On Fig. 7 it is shown the temperature increase that corresponds to the heat-dissipation coefficient $K_{h}$ increase.

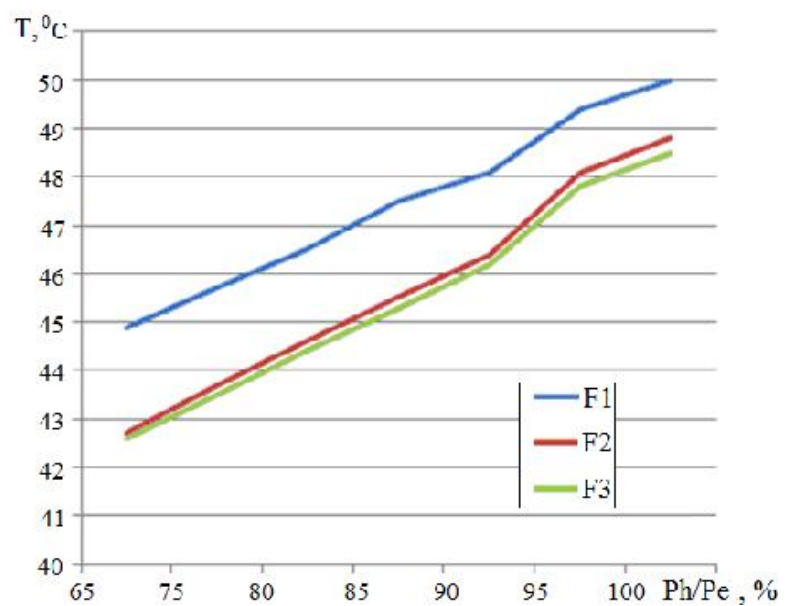

Fig. 7. Temperature of control points of the LED module in different working conditions received after simulation.

We can see clearly from the graphics that with increase of $K_{h}$ there is a sharp increase of temperatures in the control points. Given the resulting temperatures in the control points, (F1 to F3) we can make a conclusion that good thermal efficiency can be observed in the cases of values from 0.65 to 0.90 . For optimal working variant we can assume $K_{h}=0.90$, for which the temperature of the LED structure keeps low enough while providing enough power. In this way the reliability and continuous work of the LED module as well as good lighting characteristics are provided. 
In the practical operation conditions, the relationship between heat-dissipation coefficient $K_{h}$ and the junction temperature $T_{j}$ for constant $P_{d c o n s t}$ is fairly linear. Therefore, it can be approximated $K_{h}$ as a linear relationship, where the coefficients $a$ and $b$ in the right side of the (6) can be calculated for $P_{d c o n s t}$, i.e. $P_{d c o n s t}$ is a parameter in given (6)

$$
K_{h}\left(T_{j}, P_{d}\right)=a T_{j}+b
$$

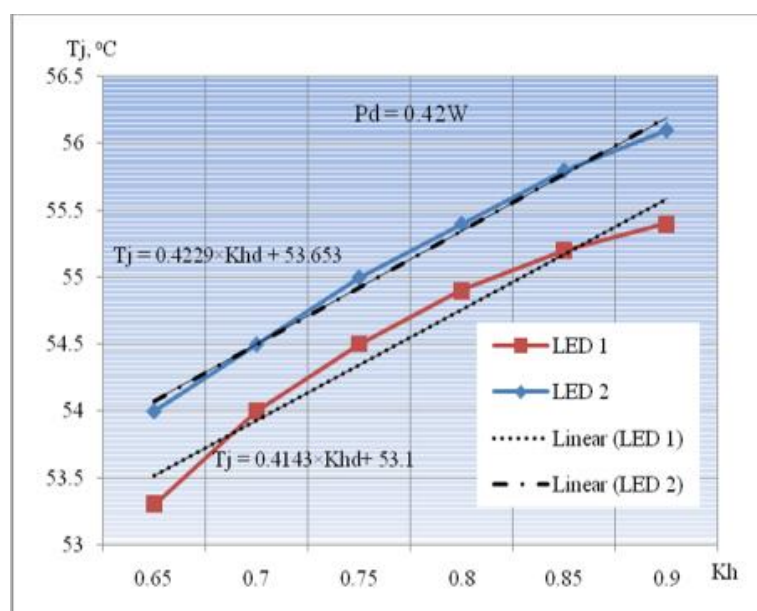

Fig. 8. Curves of $T_{j}$ vs $K_{h}$ after CFD simulations and corresponding linear approximations.

Simulated results for two different designs of LED modules with Luxeon REBEL White $1 \mathrm{~W}$ diode and corresponding linear approximations as graphs and equations, respectively, are shown in Fig. 8. The LED2 module with a $2 \mathrm{~mm}$ wider printed metal layer displays a junction temperature lower with $1,2{ }^{\circ} \mathrm{C}$ for $K_{h}=0.75$. The temperature difference is increased to $3^{\circ} \mathrm{C}$ at $P_{d, \max }$ and the same $K_{h}$.

\section{CONCLUSIONS}

In the presented research we did a practical evaluation of the heat efficiency of LED module Luxeon REBEL White $1 \mathrm{~W}$ in different working conditions. A detailed analysis for thermal modes is performed, using physical experiment with IR-camera and measured results are compared with results from thermal simulation for better results evaluation. A new approach is presented for heat efficiency estimation of LED modules for given working conditions. From the acquired results during thermal simulations we concluded that optimal working conditions of the examined LED module lies in the range from $65 \%(0.273 \mathrm{~W})$ to $90 \%(0.378 \mathrm{~W})$. The design is optimized for continuous and reliable performance of the LED module up to $90 \%$ of nominal power, even with higher environment temperatures by using the new approach.

\section{ACKNOWLEDGMENT}

We thank Octa Light PLC for their assistance in research.

\section{REFERENCES}

[1] Energy conversion efficiency by LED's used in solid state lighting. [Online]. Available: http://secondlawoflife.wordpress.com/2010/01/ 01/energy-conversion-efficiency-by-pv-cells-and-led\%E2\%80\%99s/

[2] F. Schubert, Light-Emitting Diodes, Cambridge University Press: Cambridge, 2006. [Online]. Available: http://dx.doi.org/10.1017/ CBO9780511790546

[3] A. Keppens, "Modelling and evaluation of high-power light-emitting diodes for general lighting", $P h D$ Thesis, Katholieke Universitei Leuven, 2010

[4] Comparing White Light LEDs to Conventional Light Sources. [Online]. Available: http://www.cool.conservation-us.org/byorg/usdoe/comparing_white_leds.pdf

[5] O. Tetervenoks, I. Galkin, "Assessment of light fluctuations of LED lampat different pulse mode regulation methods", Elektronika ir elektrotechnika, vol. 20, no. 6, pp. 42-45, 2014. [Online]. Available: http://dx.doi.org/10.5755/j01.eee.20.6.7265

[6] Comparing White Light LEDs. [Online]. Available: http://www.iald.org/userfiles/file/PDFs/DOESSLMaterials/04_compa ring_white_leds.pdf

[7] J. Son, B. Kim, C. Ryu, Y. Song, H. Lee, J. Choi, J. Lee1, "Enhancement of wall-plug efficiency in vertical InGaN/GaN LEDs by improved current spreading", Optics express, vol. 20, no. S2, 2012. [Online]. Available: http://dx.doi.org/10.1364/OE.20.00A287

[8] M. Kruger, "Alternative calculation method will improve therma analysis of LEDs", Philips Lumileds white paper. [Online]. Available: http://www.fortronic.it/user/file/A\%26VElettronica/Alternative\%20 Calculation\%20Method\%20Will\%20Improve\%20Thermal\%20Analy sis\%20of\%20LEDs.pdf

[9] H. Ryu, "Analysis on the luminous efficiency of phosphor-conversion white light-emitting diode", Journal of the Optical Society of Korea, vol. 17, no. 1, pp. 22-26, 2013. [Online]. Available: http://dx.doi.org/10.3807/JOSK.2013.17.1.022

[10] A. Andonova, "LED PCB thermal simulation using FLOEFD", Eastern-European Journal of Enterprize Technologies, vol. 6, no. 11, pp. 59-61, 2012.

[11] A. Andonova, G. Angelov, Y. Georgiev, T. Takov, "Application of IRT NDT for ensuring heat robustness of LED Modules", in Proc. 29th Intl. Conf. Microel. (MIEL 2014), May, Belgrade, Serbia, 2014, pp. 303-306. [Online]. Available: http://dx.doi.org/10.1109/ miel.2014.6842149

[12] A. Andonova, A. Radev, K. Stankulov, „Accelerated aging for LEDs“, Annual Journal of Electronics, vol. 2, pp. 55-58, 2012.

[13] T. Chenm H. Tao, R. Hui, „Estimation of optical power and heatdissipation coefficient for the photo-electro-thermal theory for LED systems“, IEEE Trans. Power Electronics, vol. 27, no. 4, pp. 2176 2183, 2012. [Online]. Available: http://dx.doi.org/10.1109/TPEL. 2011.2165736

[14] LUXEON rebel and LUXEON rebel ES colors. [Online]. Available: http://www.philipslumileds.com/products/luxeon-rebel/luxeon-rebelwhite

[15] K. Elissa (unpublished). [Online]. Available: http://www.flir.com/ uploadedFiles/Thermography_APAC/Products/Product_Literture/SC6 40_Datasheet\%20APAC.pdf

[16] FloTHERM Suite. [Online]. Available: http://www.mentor.com /products/mechanical/flotherm/flotherm/technical-specifications

[17] LUXEON Rebel Plaform Thermal measurement guidelines. [Online]. Available: Luxeon LED Thermal Measurement Guidelines.pdf 